\title{
MASER LINE PROFILES AND MASS OUTFLOW OF LONG-PERIOD VARIABLE STARS
}

Friso M. Olnon

Max-Planck-Institut für Radioastronomie

5300 Bonn, FRG

The long-period variable giants with maser emission (maser /LPV stars) can be divided into two groups. The stars in group 1 have color indices $I(0.84 \mu)-K$ $(2.2 \mu)<6$, those in group 2 have $I-k \geq 6$. Fig. 1 gives the velocity structure of the maser lines for both groups. I have included only those maser /LPV stars for which the radial velocity of the star is known. In most cases this is the mean velocity of the outer $\mathrm{OH}$ peaks (Winnberg, this Colloquium), but for $\mathrm{R}$ Leo and $\mathrm{R}$ Cas the stellar velocity is found from the ground-vibrational state sio emission (Reid and Dickinson, 1976). The abscissa of fig. I gives the velocity relative to the stellar velocity and the verticale scale gives the number of stars that show emission in a specific line at the relative velocity indicated. Note that the "line profiles" obtained in this way are not real mean profiles, because I only counted the presence of emission, weak or strong.
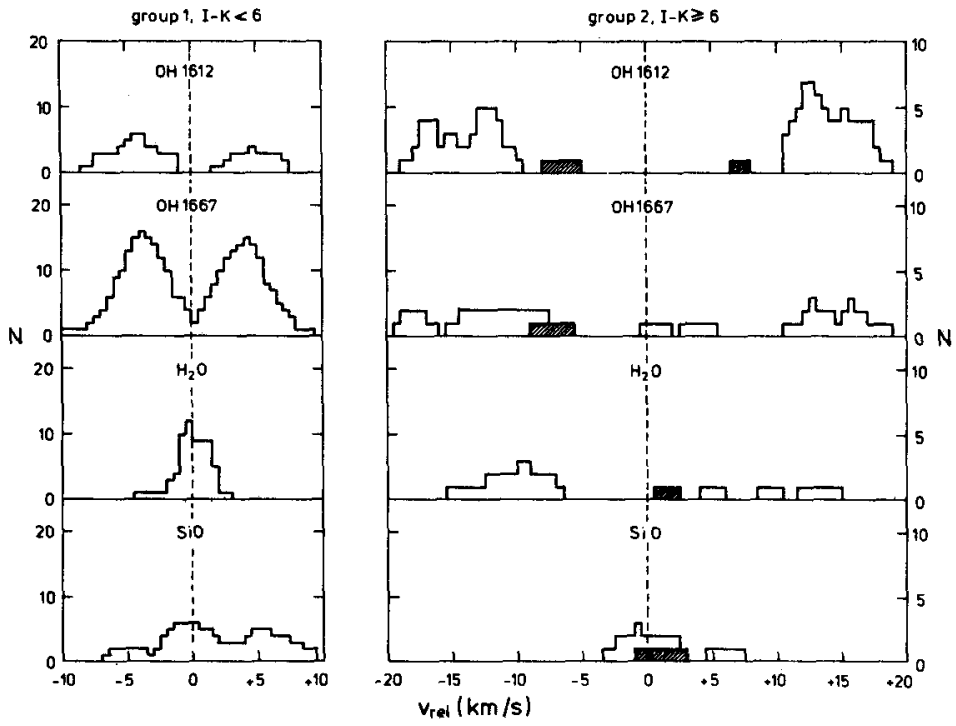

Fig. 1. The velocity structure of the maser emission for the two groups of giant maser /LPV stars. The contributions of WX Ser $(1-K=6.1)$ to the histograms of group 2 are shaded. 
The characteristics of the $\mathrm{OH}$ emission are well-known: (i) For stars with high $\mathrm{I}$ - $\mathrm{K}$ indices the $1612 \mathrm{IHz}$ line is stronger than the $1667 \mathrm{MHz}$ line (type $\mathrm{IIb} \mathrm{OH}$ emission), while the $1667 \mathrm{MHz}$ line is the stronger for stars with lower color indices (type $I \mathrm{OH}$ emission). In fig. 1 this shows up as a higher detection rate for $1612 \mathrm{MHz}$ emission in group 2 and for $1667 \mathrm{MHz}$ emission in group I. (ii) The 1667 $\mathrm{MHz}$ and $1612 \mathrm{MHz}$ features are found at the same velocities. (iii) The total velocity range, or peak separation, of the $\mathrm{OH}$ emission in group 2 is about twice as large as that in group 1 . The different $\mathrm{H}_{2} \mathrm{O}$ properties of the group have not been mentioned before. In group 1 the $\mathrm{H}_{2} \mathrm{O}$ emission is concentrated around the stellar velocity, while in group 2 it occurs at relative velocities of about $10 \mathrm{~km} / \mathrm{s}$, close to the $\mathrm{OH}$ peak velocities. A recent version of fig. 1 , which includes new sensitive $\mathrm{H}_{2} \mathrm{O}$ results, is far more convincing than the figure given here. The double-structure in $\mathrm{H}_{2} \mathrm{O}$ for group 2 stars was also found by Kleinmann and Dickinson (1976). The structure of the Sio lines appears to be the same for both groups. The emission is centered around the stellar velocity with a slightly higher probability for emission at positive relative velocities than at negative velocities. Again, more recent Sio measurements strengthen this conclusion.

What do the line profiles tell us about the velocity fields in the masing regions? Let us assume that population inversion between the maser levels, and hence maser amplification, occurs within a fairly thin spherically symmetrical shell of the stellar envelope. Emission at some velocity $v_{z}$ can only arise from molecules that move with a line-of-sight velocity $v_{z}$ and this emission can only be amplified by molecules along the same line of sight with velocities in the interval $v_{z} \pm 0.5 v_{t h}$, where $v_{t h}$ is the thermal velocity. Thus, maser emission at $v_{z}$ originates in a ring bounded by the isotaches (planes of equal line-of-sight velocity) $v_{z} \pm 0.5 v_{t h}$ and the boundaries of the shell. Fig. 2 shows a cross-cut of the shell and the ring. The 1 ine intensity at $v_{z}$ is essentially determined by the (negative) optical depth in the masing transition along the line of sight through the $v_{z}$ ring. In a homogeneous shell the optical depth is proportional to the geometrical pathlength through the ring, the coherence length. The maser line will reach its peak intensity at the velocity where the coherence length is maxima?.

Fig. 3 gives the isotaches for three different velocity laws in the maser shell, and the corresponding line profiles. Of course, the profiles depend on the thickness of the shell and the ratio of thermal velocity and flow velocity. For instance, if the shell is very thin or the velocity ratio very high, the longest coherence length occurs at $v_{z}=0$, independent at the velocity field. We then find a single peak at the stellar velocity (tangential maser action). 


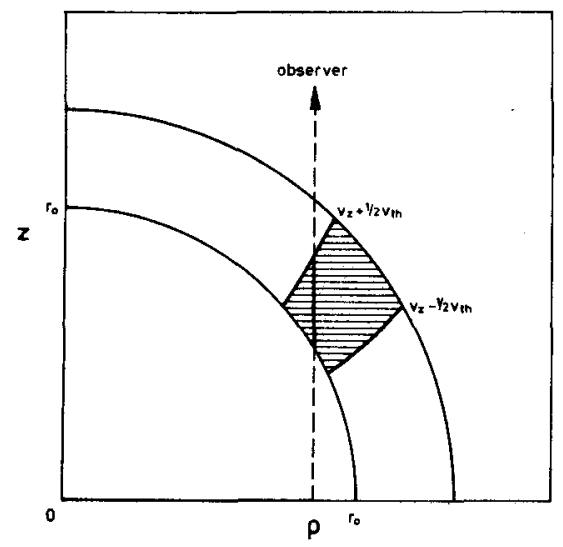

Fig. 2. A cross-cut of the maser she11. The hatched region is a slice of the ring where the emission observed at velocity $v_{z}$ comes from. The region is bounded by two isotaches labeled with their line-ofsight velocities. The thick line segment is the coherence length for emission at $v_{z}$ along the dashed line of sight.

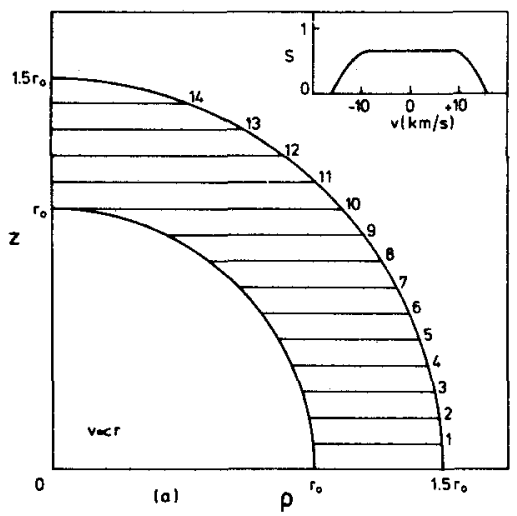

Fig. 3. The isotaches within a crosscut of the maser shell for three different velocity laws: (a) $v a r$, (b) $v=$ constant, and (c) $v a l / r$. In all three cases the flow velocity at the inner radius of the shell is 10 $\mathrm{km} / \mathrm{s}$. The isotaches are labeled with their line-of-sight velocities in $\mathrm{km} / \mathrm{s}$. In the upper right corners of the figures schematic line profiles are drawn.
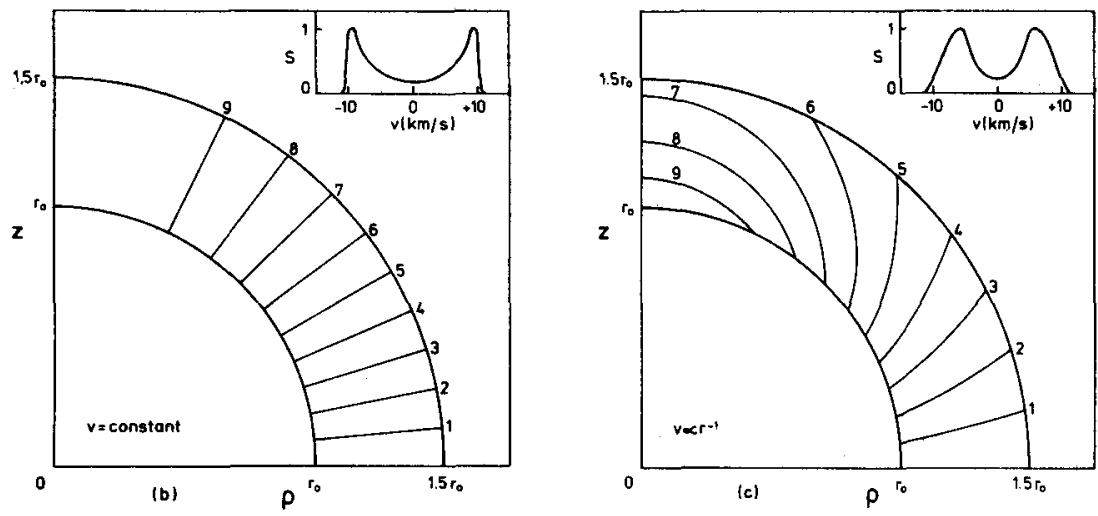


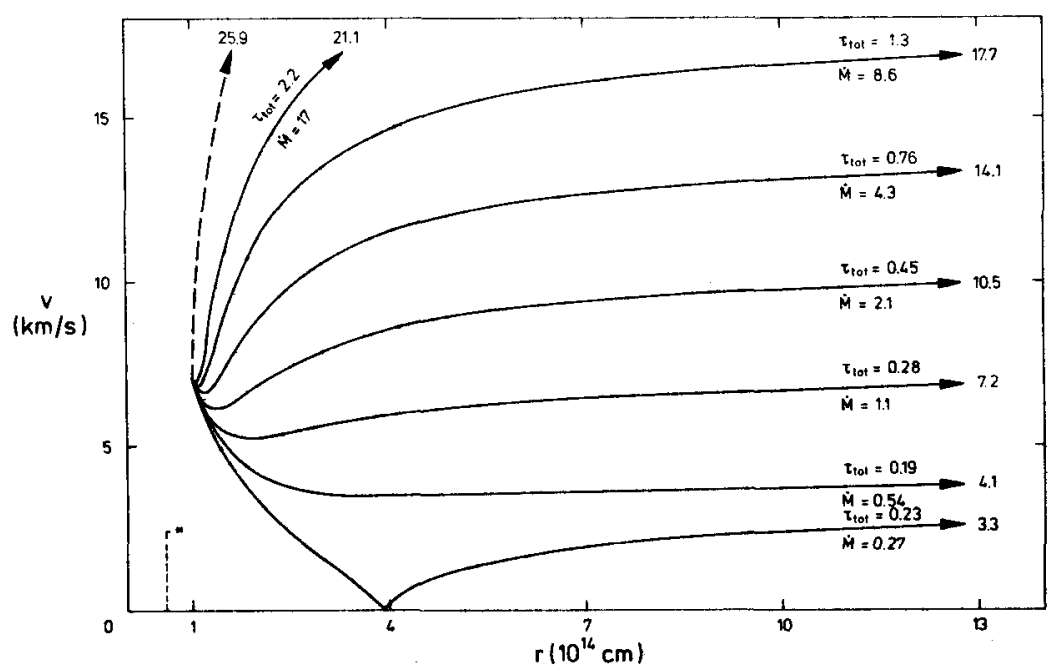

Fig.4. The flow velocity, $v$, as a function of distance, $r$, for some model envelopes. Along each curve are given the corresponding values of the total optical depth for direct stellar radiation, $\tau_{\text {tot, }}$ and the mass loss rate, $\dot{M}$ in units of $10^{-5} \mathrm{M}_{\Theta} / \mathrm{yr}$. The numbers at the end of each curve give the terminal flow velocity in $\mathrm{km} / \mathrm{s}$. The dashed curve refers to the 1 imit of very large optical depth and mass loss rate.

In fig. 4 I show the velocity curves for some model envelopes. I mention only briefly the properties of the models; the details will be published elsewhere. The central stars are typical oxygen-rich Miras that are losing mass at a rate $M$ given for each curve. Somehow, the flow reaches a distance of $10^{14} \mathrm{~cm}$ with a flow velocity of $7 \mathrm{~km} / \mathrm{s}$. At that point silicate particles condense and the radiation pressure on these grains determines the velocity at larger distances. In the dust envelope the stellar radiation is partly absorbed and the energy is reradiated at longer wavelengths, where the radiation pressure efficiency of the silicates is higher. The "reddening" of the radiation flux is stronger for higher mass loss rates. Thus, stars with high mass loss rates have high $I-k$ indices and large flow velocities (group 2 properties) as compared with low-mass-loss stars (group 1).

The flow velocity approaches its terminal value at about 10 stellar radii. Maser emission from larger distances will always show two peaks (fig. 3b) and the 
line profiles are independent of the precise distance. The VLBI results of giant maser /LPV stars indicate that the $1612 \mathrm{MHz}$ OH emission originates typically at 100 stellar radii, and the $1667 \mathrm{MHz}$ emission at somewhat smaller distances from the star. In both lines half the peak separation is equal to the terminal flow velocity; say $6 \mathrm{~km} / \mathrm{s}$ for group 1 and $15 \mathrm{~km} / \mathrm{s}$ for group 2 stars.

The $\mathrm{H}_{2} \mathrm{O}$ maser region must be quite close to the stellar surface, because the maser levels lie high above the ground state. On the other hand, it cannot be too close, because then the gas density and temperature become so high that collisions thermalize the level populations. The single-peak $\mathrm{H}_{2} \mathrm{O}$ emission of group 1 stars can only be produced by tangential maser action. Therefore, the maser shell must be very thin and the thermal velocity quite high: the $\mathrm{H}_{2} \mathrm{O}$ line is probably formed in the innermost region of the envelope at about 1.5 stellar radii. For group 2 stars the gas density. at the distance is too high due to the higher mass loss rate, and population inversion is only possible at larger distances. There, the flow velocity is higher and the thermal velocity lower: tangential maser action is no longer dominant and a double-peaked line appears. The group $2 \mathrm{H}_{2} \mathrm{O}$ peaks have definitely a lower relative velocity than the $\mathrm{OH}$ peaks. From fig. $4 \mathrm{I}$ conclude that the $\mathrm{H}_{2} \mathrm{O}$ maser shell has a radius of about 3 stellar radii.

The Si0 maser levels 1 ie even higher above the ground state than those of $\mathrm{H}_{2} \mathrm{O}$, and the SiO abundance drops sharply as soon as silicate particles condense. The Si0 maser lines must come from the dust-free inner region of the envelope. The line profiles, and especially the time variations, might tell us a lot about the conditions in the innermost layers of the envelopes and, thereby, about the mass loss mechanism in LPV stars.

\section{REFERENCES}

Kleinmann, S. G., Dickinson, D. F. 1976, Bul1. Am. Astron. Soc. 8, 552. Reid, M. J., Dickinson, D. F. 1976, Astrophys. J. 209, 505.

\section{DISCUSSION of the paper by OLNON:}

SURDEJ: How did you solve the radiative transfer in the envelope where maser line profiles arise?

OLNON: I did not calculate the radiative transfer. I simply assumed that the total flux is distributed over two blackbody components, one at the stellar temperature and the other at some envelope temperature. With increasing distance, thus increasing optical depth for stellar radiation, more and more of the flux is contained in the cooler component. That fraction depends on the mass loss rate and the velocity field. 